\title{
Methodological Reflection on an Initiated Expert Stakeholder Engagement Process. A Case Study of a Dementia Prediction Discourse
}

Julia Perry ( $\sim$ julia.perry@medizin.uni-goettingen.de)

\section{Research}

Keywords: Stakeholders, participation, discourse, evaluation criteria, health care policy making, dementia prediction, Germany

Posted Date: December 10th, 2020

DOI: https://doi.org/10.21203/rs.3.rs-123519/v1

License: (c) (1) This work is licensed under a Creative Commons Attribution 4.0 International License.

Read Full License 


\section{Abstract}

\section{Background}

Engagement and participation are vital for including experiences, opinions, or expertise regarding the need for action and specifying goals of relevant stakeholders. In recent years, engagement processes have significantly gained in prominence especially in the field of healthcare; and participation has been actively demanded by public health agencies as well as by health research funding organizations. Despite numerous international work and wide agreement on the benefits of stakeholder engagement, there is still limited published empirical evidence on methodological best practices for stakeholder approaches and even less on evaluation or outcomes in the process of developing policy.

\section{Methods}

On the basis of previous research on stakeholder discourses and with the German case study at hand, I inferred normative criteria for the evaluation of discourse processes. For the methodological examination, my manuscript presents a comprehensive framework of normative criteria relevant to stakeholder engagement planning, implementation, evaluation and reporting, and to illustrate different evaluation and reporting needs for research on stakeholder engagement, namely, depth of participation, legitimate selection of stakeholders, representativeness, process management, output, role definition, and transparency.

\section{Results}

The developed normative evaluation criteria make the evaluation of an engagement process with a large amount of material possible. My analysis indicates the key facilitating elements and also barriers of this participatory approach, demonstrating the importance of especially deploying the following evaluation criteria: output, representativeness, process management, and transparency.

\section{Conclusion}

The seven developed evaluation criteria have been effective for the evaluation of the stakeholder discourse on dementia prediction. These evaluation criteria may also be conducive to the evaluation of engagement processes on other health research topics with a large corpus of material. To date and to my knowledge, a comprehensive evaluation framework for engagement processes with experts in the field of assessing biomedical issues is missing in the literature and my work aims at bridging this gap.

\section{Introduction}

Current news headlines promise new blood tests that may predict Alzheimer's disease[1]. The articles not only raise public awareness about potential testing options but also about potential negative effects and challenges, for example, not having a cure in place. 
In the past, there have been various topics on emerging medical technologies or scientific breakthroughs that have raised public controversies (Whitty 2013). Some of these were addressed with public engagement and patient and public involvement (PPI) approaches, for example, in the fields of stem cell, cancer research, or biobanks (Adashi et al. 2019; Pii et al. 2019; Hansen et al. 2018; Barfoot et al. 2017; O’Doherty \& Burgess 2013).

To date, Germany has not specifically focused on implications of dementia prediction. With an increasing focus on research in the field of biomarker[2]-based blood tests with high sensitivity and specificity for the prediction of Alzheimer's dementia (Nabers et al. 2019), from a medical-ethical and social science perspective, the scientific and moral necessity arose to take up the discussion in the German-speaking context.

Therefore, we initiated an interdisciplinary, nation-wide stakeholder discourse. Our aim was to raise questions regarding what ethical, social, and legal implication dementia prediction entails and specifically, what frameworks are necessary in Germany before such tests are implemented in clinical practice. Previous research has shown that forms of bottom-up engagement with the aim of policy development can frame important perspectives, especially with outcome-focused approaches in new, complex, and uncertain fields (Atkins 2012; MacLean \& Burgess 2010).

This paper offers a critical methodological reflection of the chosen stakeholder discourse procedure. To date, there is not much experience especially in the context of Germany. The analysis of this discourse approach on implications of dementia prediction can serve as case study as the discussion and methodological reflection of engagement approaches is still in its infancy.

The topic of dementia prediction differs from other controversial topics such as, for example, breast cancer. Dementia, due to the aspect of cognitive impairment, has for a long time stood as a barrier to any form of participation including the exclusion from research participation (Jongsma et al. 2016). Thus, the significance of including a stakeholder group in the context of dementia prediction is very high.

The guiding research question of this paper is: What criteria are important for systematically evaluating a stakeholder approach with the intention of policy-oriented outcomes?

The aim is to expand and ameliorate existing criteria previously considered for the purpose of evaluation of discursive stakeholder approaches. Hence, the objective of this article is to methodologically examine and reflect on stakeholder engagement for obtaining and integrating informed stakeholder voices into a policy-making process.

This paper focuses on developing a comprehensive framework of normative criteria relevant to stakeholder engagement planning, implementation, evaluation and reporting, and to illustrate different evaluation and reporting needs for research on stakeholder engagement. The objective of this study is firstly, to analyze how participation of stakeholders can support policy-oriented discussions on a critical 
issue such as dementia prediction. Secondly, how the concepts of involvement and participation can be evaluated systematically with a normative evaluation framework.

\section{Background}

\section{Developments and implications of dementia prediction}

Due to the prevalence and severity of dementia and the current lack of effective treatments, the disease has been made a global health priority. The expected number of people with dementia in the future will further put pressure on health care systems (WHO 2017). Accordingly, there has been a shift towards the implementation of public dementia (prevention) strategies as well as pre-clinical/symptom-free risk profiling prior to the development of actual symptoms (Leibing \& Schicktanz 2021). Numerous researchers have pointed to the need for exploring various biomarkers for the purpose of dementia prediction and early detection (Nabers et al. 2019; Márquez \& Yassa 2019). However, it remains unclear whether one or multiple biomarkers actually will offer a reliable indicator for the onset or progression of dementia (Glymour et al. 2018). Nevertheless, such predictive tests will undoubtedly be applied in clinical practice in the near future. The clinical application of such tests for people without objective symptoms, however, entail unresolved ethical, legal, and social issues that should be addressed in advance (SchmitzLuhn et al. 2019; Milne et al. 2018).

The potential advantages of dementia prediction include more effective pharmaceutical research, earlier detection, and potentially earlier administration of treatment options as well as the ability of affected individuals to plan potential care and living arrangements. Possible problems lie in the mental burden of anticipating (potentially in error) the onset of dementia as well as in forms of social exclusion, as no cure currently exists. Additionally, since test results are expressed as notoriously non-intuitive risk probabilities, informed consent and patient education become especially challenging (Alpinar-Sencan et al. 2019; Mozersky et al. 2018).

Hence, current developments involving biomarkers for dementia prediction allow timely reflection of the ethical, legal, and social implications, the political frameworks, and societal strategies needed to improve public and clinical communication about early dementia and dementia research. One important, ethically motivated approach is to enable a participatory, discourse approach with relevant stakeholders in the field of dementia and dementia prediction. The issue is highly relevant for the general public due to the unresolved conflicts arising for individuals and society as a whole. For socially acceptable and comprehensive policy development and recommendations in this context, it is not sufficient to solely involve established political bodies as active decision-makers. These bodies are often made up of medical experts. For example, patient advocacy experts are needed as well as a broad spectrum of other stakeholders to contribute to the discourse. Stakeholders and participation are here examined jointly under the focus of stakeholder participation as a sub-theme of public involvement.

\section{The relevance of engaging and participating in health care issues}


While an urgent need for engagement is expressed, there is a great variation in depths of engagement ranging from information, consultation to participation (Brown \& Bahri 2019; Deverka et al. 2013). Often there is a lack of precise conceptualization and definition of what engagement means and entails. Terms such as 'engagement', 'participation', and 'involvement' have been applied indistinctly and interchangeably in current literature adding to an inflationary or even tokenistic use of participation rhetoric without a critical reflection and little guidance on how to engage relevant or affected groups in a meaningful manner (Brown \& Bahri 2019; Pollock et al. 2018; Boaz et al. 2018; Ray \& Miller 2017).

Engagement and participation have significantly gained in prominence in the field of healthcare over the past years (Pii et al. 2019; Kim et al. 2018; Souliotis 2016). Participation has been actively demanded by public health agencies such as the World Health Organization (WHO 2013) as well as health research funding organizations (Knoepke et al. 2019; Boaz et al. 2018). Patient organizations and advocacy groups are increasingly raising their voices to be included in policy-oriented decisions (Manafo et al. 2018; Schicktanz 2015).

Some research on participation in the health context focuses on an asymmetrical form of participation with people or organizations within the healthcare system granting those outside access to or a voice in matters of health (Potvin 2007). Other frameworks of participation are structured more inclusively. They involve stakeholders from various services, for example, making a continuous exchange of knowledge possible and moving affected interests forward (Slack et al. 2018; Brereton et al. 2017; Lemke \& HarrisWai 2015). While applied in fields of uncertainty or conflicts in technology and science, participation can encourage certainty by acquiring knowledge and experience and exchanging knowledge for the purpose of creating shared understanding (Brown \& Bahri 2019; Potvin 2007). Further, PPI is considered imperative for the identification of relevant foci in treatment and research (Pii et al. 2019; Sacristán et al. 2016). Participation as an approach aims to be democratic, empowering, and educational (Pii et al. 2019; Beier et al. 2016; Banks et al. 2013: MacLean \& Burgess 2010).

\section{Involving stakeholders in health policy discourses}

For my working definition, stakeholder participation can be described as the participation of a group of specified people, those who have an interest in an issue, and also as one form of participation in the broader spectrum of participation.

Stakeholders include people, groups, organizations, and associations at local, national, or international level who are affected by social decisions and who are entitled to be considered in these decisions (Lemke \& Harris-Wai 2015). Stakeholders may have a direct interest in the process or outcomes of a project, research or policy issue (Boaz et al. 2018; Brereton et al. 2017; Deverka et al. 2013). Stakeholders can bring in important experiences, opinions, or expertise regarding the need for action and specifying goals (Murtagh et al. 2017; Brereton et al. 2017; Concannon et al. 2014; Deverka et al. 2013).

Stakeholders therefore can assume a normative right to participation (Hansen et al. 2018). However, it is anticipated that the interests of various stakeholders can also differ and that stakeholders can differ in terms of their influence, authenticity, and legitimacy (e.g. Boaz et al. 2018; Agné et al. 2015; Kelty et al. 
2015; MacLean \& Burgess 2010). A balanced composition of various stakeholder representatives in a discursive process is therefore vital. In a multidisciplinary discourse, it is not just a question of understanding who is affected by decisions, but also of who can and should actually be able to influence their impact. A discourse is often a political space that is not yet fully regulated, especially in the case of dementia prediction, as guidelines for the clinical implementation of the new biomarker technology are still lacking. The interest in engaging stakeholders more broadly grew from an aspiration to deal with "the lack of real-world relevance of research and to ensure more effective implementation of research findings into practice" (Pollock et al. 2018).

Germany, as a country with a publicly-funded health care system based on the principle of solidarity, has rarely engaged and promoted participation of the public, patients, and other stakeholders, especially in contrast to other industrialized countries such as the UK, North America, and Australia (Pii et al. 2019). Thus, Germany can be considered rather new to such engagement discourses. This could be explained by the established structure of medicine and the healthcare system in Germany guided by functional and professional self-regulation (Schicktanz 2008).

While bioethical topics have been addressed in discourse procedures, for example on genetic diagnostics (https://www.springer.com/de/book/9783810036292), stem cell research (https://www.gen-ethischesnetzwerk.de/diskursoffensiven; http://imgb.de/Projekte/ClinhiPS/) or brain research (https://www.jura.uni-bonn.de/centre-for-the-law-of-life-sciences/forschung/) in Germany, no discourse procedure has been carried out in Germany or internationally previous to ours that primarily dealt with (early) diagnosis of dementia or other research in the context of dementia.

Further, despite numerous international work on and wide agreement on the benefits of stakeholder engagement, there is limited published empirical evidence on methodological best practices for stakeholder approaches and even less on evaluation or outcomes in the process of developing policy (Laird et al. 2020; Balane et al. 2020; Ray \& Miller 2017; Lemke \& Harris-Wai 2015). As Goodman and Thompson stress there is a critical difference between "going through the empty ritual of obtaining stakeholder feedback and giving stakeholders the real power needed to affect the research process and resulting outcomes" (2017).

\section{Design and study setting: case study}

This paper aims at inducing optimized normative evaluation criteria for engagement processes. In the following, our discourse procedure on dementia prediction is described. It serves as a case study for developing normative evaluation criteria and a framework for the evaluation of future engagement processes.

In this project, I was employed as a research associate and was responsible for preparing scientific research on the topic and supporting in all necessary steps for conducting the project. 


\section{Iterative process of engaging stakeholders and collection of participation elements}

We based the participatory discourse on information about dementia prediction from current literature and research. This information - which formed the starting point for the research project and first made it possible to initiate and carry out this stakeholder approach - also frames the ethical premises of the discourse. These premises include that resources as well as ethical, social, and legal recommendations for future consulting professions are currently missing in the field of dementia prediction and need to be developed, as counseling professionals will increasingly be asked to provide advice and support for people seeking advice about predictive testing, in the near future.

As a four-headed team in the research project[1], we initiated the stakeholder discourse guided by our interdisciplinary project advisory board consisting of seven external members. The stakeholder engagement activities occurred over 24 months. The documented material of the activities is described in Figure 1.

First, we identified those organizations and associations that deal with issues of health in a broader sense and with dementia specifically, including areas such as counseling, care, research, patient advocacy, policy making, governance, financing and insuring, scientific networks, welfare, religion, and ethics (see Figure 2 for fields of participating organizations and associations). Thus, the aim was to identify all organizations and associations that had a stake in policy development or the development of recommendations on issues of dementia prediction, dementia care, and dementia research in the German context.

80 organizations and associations (see Supplemental Material 1 for full list) were initially identified in an iterative procedure, supported by our experienced advisory board. 75 of those organizations were contacted on board level with an invitation to participate in the discourse. The contacted person was then able to delegate specific people within their organization or association to answer the posed questions and one person to volunteer as a representative at the stakeholder conference which took place in Göttingen, Germany in 2018.

The one-page invitation for participation in the discourse procedure included brief information on the background and aims of the project as well as an appeal to the organization's participation. Further, the organizations were asked to answer the following questions on an approx.one-page position paper which was sent by post and by email to allow for an easy and time-efficient response. The first question was asked to contextualize their statement and also raise the organizations' awareness to where ethical, legal, or social issues could arise in the context of dementia prediction.

A. What is the main interest in dealing with dementia in your organization?

B. Please outline your central position / view for predicting dementia using biomarkers. Where do you see key issues or controversies? 
The involvement of different stakeholders in a procedure may entail different levels of medical knowledge, affectedness or sometimes conflicts of interest. As complex, technical information often plays a role in the deliberation of bioethical questions, the project team was concerned to address potential imbalances in the discussion and to enable stakeholders to have as equal access as possible to terminology and developments in research. The aim in the procedure was to enable a discourse that is as free from hierarchy, inclusive and comprehensive. That is why the background materials were sent to the participating stakeholders in advance.

Of these 75 addressed organizations and associations, 29 reported back to us with a position paper. In the meantime, the German Medical Association published a topical statement independently of the discourse procedure, which was also included in the discourse. 25 of the organizations nominated delegates to participate in the two-day intensive conference with the aim of producing a joint statement. Finally, 24 stakeholders attended the stakeholder conference held in June 2018 at which a joint statement was produced.

The final version of the joint statement was published at the end of 2018. It was sent to all initially identified organizations and participating stakeholders for commenting. This initiated commentary phase allowed the participating organizations to address points that they were unable to present in a more differentiated or nuanced manner in the joint statement due to the consensus process. Therefore, the commentary phase was essential for opening room for plural perspectives. 12 organizations made use of the opportunity to comment within the given period of time, four of which had not previously submitted their own statement or participated in the stakeholder conference.

In all, 33 organizations and associations (see Supplemental Material 1) actively participated in the discourse process at various stages. Due to the iterative, multi-stage procedure developed in the research project for discursive participation, stakeholders could engage in up to four successive phases: provision of written input, conference participation, commenting of joint statement and for means of being informed about the discourse (see Figure 1). For further details on the participating organizations see Supplemental Material 1.

\section{Methodology}

As the aim of this paper is to develop normative evaluation criteria based on the methodological reflection on an initiated engagement process. I will describe the successive steps of my analysis in this section.

\section{Induced normative criteria for evaluating the stakeholder discourse}

Already identified shortcomings or constraints in previous engagement studies mainly include issues of representation, communication and articulation, impacts, and democracy (Murtagh et al. 2017). To date, a comprehensive evaluation framework for engagement processes with experts in the field of assessing biomedical issues is missing in the literature. 
For the methodological examination and reflection of the stakeholder discourse at hand, and for the development of a comprehensive framework of concepts relevant to stakeholder engagement planning, implementation, evaluation and reporting, the following seven normative criteria were induced: depth of participation, legitimate selection of stakeholders, representativeness, process management, output, role definition, and transparency. I composed the criteria based on existing recommendations and single findings from conducted stakeholder approaches in the literature as well as own experience gathered during the stakeholder approach at hand. Thus, the evaluation criteria listed in Table 1 can be regarded as a collection and combination of already established criteria (see column "Examples inferred from the literature") assessing different kinds of engagement processes as well as criteria developed and refined from the corpus of material of the discourse. This, to my knowledge, is the first paper bringing together established criteria and applying and amending them to an expert stakeholder discourse focusing on a bioethical topic.

Table 1: List of refined and expanded evaluation criteria 


\begin{tabular}{|c|c|c|c|}
\hline No. & Criteria & Key concepts and keywords & Examples inferred from the literature \\
\hline 1 & $\begin{array}{l}\text { Depth of } \\
\text { participation }\end{array}$ & $\begin{array}{l}\text { Information, consultation, } \\
\text { participation, involvement, } \\
\text { reflexivity, communication, } \\
\text { articulation, democracy, } \\
\text { decision-making, } \\
\text { methodological approach, } \\
\text { depth of information, } \\
\text { direction of involvement }\end{array}$ & $\begin{array}{l}\text { Archana et al. 2019; Brown \& Bahri } \\
\text { 2019; Gesell et al. 2019; Kendall et al. } \\
\text { 2018; Gesell et al. 2017; Goodman \& } \\
\text { Thompson 2017; Lemke \& Harris-Wai } \\
\text { 2015; Deverka et al. 2013; Hermans et } \\
\text { al. 2011; Burton et al. } 2009\end{array}$ \\
\hline 2 & $\begin{array}{l}\text { Legitimate } \\
\text { selection of } \\
\text { stakeholders }\end{array}$ & $\begin{array}{l}\text { Selection criteria, inclusion, } \\
\text { research focus, recruitment, } \\
\text { contact measures }\end{array}$ & $\begin{array}{l}\text { Boaz et al. 2018; Gesell et al. 2017; } \\
\text { Brereton et al. 2017; Goodman \& } \\
\text { Thompson 2017; Forsythe et al. 2016; } \\
\text { Concannon et al. 2014; Lemke \& Harris- } \\
\text { Wai } 2015\end{array}$ \\
\hline 3 & Representativeness & $\begin{array}{l}\text { Representation, inclusivity, } \\
\text { heterogeneity, minority } \\
\text { voices, diversity, } \\
\text { interdisciplinarity, } \\
\text { multidisciplinarity, openness }\end{array}$ & $\begin{array}{l}\text { Archana et al. 2019; Brown \& Bahri } \\
\text { 2019; Gesell et al. 2019; Kim et al. 2018; } \\
\text { Brereton et al. 2017; Ray \& Miller 2017; } \\
\text { Murtagh et al. 2017; Lemke \& Harris- } \\
\text { Wai 2015; Concannon et al. 2014; } \\
\text { Deverka et al. 2013; Hoffman et al. } \\
\text { 2010; Parker et al. 2007; Burton et al. } \\
\text { 2009 }\end{array}$ \\
\hline 4 & $\begin{array}{l}\text { Process } \\
\text { management }\end{array}$ & $\begin{array}{l}\text { Time, timing, resources, } \\
\text { good planning, realistic } \\
\text { planning, planning process, } \\
\text { research stage, research } \\
\text { process }\end{array}$ & $\begin{array}{l}\text { Archana et al. 2019; Gesell et al. 2019; } \\
\text { Boaz et al. 2018; Kendall et al. 2018; } \\
\text { Murtagh et al. 2017; Goodman \& } \\
\text { Thompson 2017; Lemke \& Harris-Wai } \\
\text { 2015; Parker et al. } 2007\end{array}$ \\
\hline 5 & Output & $\begin{array}{l}\text { Aim, purpose, objective, } \\
\text { outcome, product, decision- } \\
\text { making, consent, dissent, } \\
\text { communication, fears, } \\
\text { hopes, criticism, praise, } \\
\text { dissemination, expectations }\end{array}$ & $\begin{array}{l}\text { Brown \& Bahri 2019; Murtagh et al. } \\
\text { 2017; Deverka et al. 2013; Cotton et al. } \\
2000\end{array}$ \\
\hline 6 & Role definition & $\begin{array}{l}\text { Roles, moderation, influence, } \\
\text { monitoring, facilitation, } \\
\text { clarification }\end{array}$ & $\begin{array}{l}\text { Boaz et al. 2018; Kim et al. 2018; } \\
\text { Hermans et al. 2011; Hoffman et al. } \\
2010\end{array}$ \\
\hline 7 & Transparency & $\begin{array}{l}\text { Trust, honesty, openness, } \\
\text { sincerity, transparency, } \\
\text { holistic approach, } \\
\text { commitment, scientific } \\
\text { research, informative }\end{array}$ & $\begin{array}{l}\text { Camelo Castillo et al. 2019; Gesell et al. } \\
\text { 2019; Brereton et al. 2017; Gesell et al. } \\
\text { 2017; Ray \& Miller 2017; Lemke \& } \\
\text { Harris-Wai 2015; Concannon et al. } \\
\text { 2014; Burton et al. } 2009\end{array}$ \\
\hline
\end{tabular}

Examining the corpus of material (see Figure 1) with these seven developed normative criteria will allow an in-depth exploration of strengths and also constraints as well as advantages and disadvantages of this discourse approach. This normative framework guided by developed criteria aims at addressing fundamentals of expert engagement in the field of emerging medical technologies and critically reflecting on them in a systematic manner. 
For this paper, I analyzed the empirical corpus of material by means of qualitative content analysis following Renz et al. 2018 and Hsieh \& Shannon 2005.

Content analysis as a method, with wide application in health research, allows for deductions by systematically identifying specific words, themes or concepts in communicative language or written material with referral to the context of the content. After identification, inferences can be made about the presence, meaning, and relationships of certain themes or recurring issues and their underlying meaning. The process of content analysis involves a systematic method of coding and classification of written material to identify patterns and themes and can be regarded as a data reduction technique. Thus, content analysis allows for the provision of substantial insights into phenomena by means of text analysis. With the established proximity of the researcher to the text, content analysis can be regarded as a highly reliable tool due to a systematic procedure of analysis (Renz et al. 2018; Hsieh \& Shannon 2005).

For the paper at hand, the method of content analysis involved close and repetitive reading of the material to obtain immersion and a sense of the whole corpus of material, reflection on the origin and authors of the text corpus, and sequential text interpretation, as well as the development of categories and codes based on the established criteria (Renz et al. 2018; Hsieh \& Shannon 2005). The overarching theme for the analysis, is the evaluation of engagement processes. The categories were developed inductively from the material at hand and the criteria were developed iteratively guided by existing evaluating frameworks of stakeholder engagement and amended by own criteria derived from the context of this particular stakeholder engagement process and inductively from the references made in the material. The normative criteria serve as a framework for the evaluation.

For the analysis, it was necessary to define a coding scheme for the organization of the data in a comprehensible and manageable manner. The coding scheme entails keywords for each developed evaluation criteria that were identified before and during data analysis (Hsieh \& Shannon 2005; see Table 1). In the coding process material was distinguished according to internal and external input. Internal input refers to material that was developed by the project team before, during, and after the engagement process. External input refers to material developed by involved stakeholders, the project's advisory board and other organizations or individuals reporting on the engagement process at hand (see Figure 1).

\section{Results Of The Case Study}

The results are structured along the seven induced normative evaluation criteria.

The discourse project's objectives for stakeholder engagement were clear, as set out in the internal documents (see Figure 1). The topic of the discourse approach is novel and has, to date, not been discussed in the German context. A nuanced and comprehensive approach appears inevitable when addressing a new medical technology which will affect not only providers, counselors, consumers but also society as a whole. This is why an iterative approach was chosen with varying opportunities and depths of engagement (see Figure 1) embracing the widest possible inclusion of organizations as well as political entities considered stakeholders on the topic of dementia prediction. 
With the methodology described above, the developed normative evaluation criteria and related key concepts and keywords (see Table 1) were formulated to guide the analysis. In the analysis, explicit and implicit references to the normative evaluation criteria were identified in the corpus of material (see Figure 1). These were assessed and are elucidated in the following section.

\section{1) Depth of participation}

The first criterion can rather be understood as an overall description of participation in the stakeholder procedure and thus a criterion embracing all following criteria.

One uniting thrust in all material was the raised necessity to deal with the topic of dementia prediction before its clinical implementation, stressing the relevance of the project initiation and recognizing the unresolved issues needing public and scientific attention. Scientific publications called the discourse approach a sophisticated and elaborate procedure which addressed a wide spectrum of participants and of engagement options over an extended period of time.

In a separate step, apart from the stakeholder conference, with the aim of broadening the spectrum and recipients of engagement, a social media strategy[1] was implemented by the project team which also allowed the public to participate on the topic debate. The aim was to create a discursive platform on which questions were posed to Facebook and Twitter users on ethical, social, and legal aspects of dementia prediction. Further, an essential aspect of the discourse was to engage future counseling professions. This was pursued in subsequently conducted seminars and workshops and the sustainable development of teaching materials.

While a wide range of stakeholders was addressed and invited to take part in the discourse and, no organization with a government mandate actively participated in the discourse by means of a position paper. Only at the podium discussion following the stakeholder conference did Lower Saxony's Minister for Social Affairs, Health and Equality highlight the importance of such a conference to bring everyone to the table and to incorporate different perspectives.

\section{2) Legitimate selection of stakeholders}

From the project's self-evaluation it became clear that the process of identifying potential stakeholders was a highly iterative one and an important step for the entire project. With the phase of thorough research on relevant organizations in Germany and the creation of an extensive list of the initially identified organizations, a first step was initiated to open a wide selection of stakeholders. This process was accompanied by a critical examination of the list by colleagues and acquaintances who are scholars in the field of dementia and could act as multipliers or mediators in the process of identification. In a subsequent step, the list was proposed to the project's advisory board. We asked the members of the advisory board to examine the list carefully for necessary additions or alterations. In this process the aim 
was to achieve a balanced representation of fields, political parity, and few exclusive experts who, for example, had previous experience in committee work.

Based on the state of knowledge of previously successfully conducted discourses on bioethical topics and after consultation with the project's advisory board, the project team decided to activate only those organizations and institutions that claim to represent social interests or have a relevant governmental regulatory mandate to participate. A meaningful participation procedure including individuals as experts would have overstrained the logistical capacities of the project and risked bias of individual interests or ties being put forward in the process that cannot be justified. However, due to pragmatic reasons, we had to expand the selection to some extent, as two organizations suggested individuals who did not act as named representatives of the organization. This led to the creation of different levels of representation in two cases where organizations were less monolithic and rather heterogenous regarding the organization's structure and thematic perspective.

Further, the project team made clear in its summaries, protocols, and its self-evaluation of the project that the identified organizations should submit a position paper from their organization's perspective which served as a condition for participation in the stakeholder conference. This aimed at identifying how the respective organization perceived its own stake in the discourse topic and which issues were most relevant from their perspective. Here there was also space for participants to formulate demands and expectations or wishes to the stakeholder conference, the entire discourse or further discourses in the future. Obtaining the position papers required immense communication coordination and perseverance on our side with continuous contact by post, email, and telephone.

After 29 position papers were submitted and carefully examined concerning the content, those organizations were invited to take part in the stakeholder conference who had expressed their willingness to participation and had named a concrete representative. The strategy of early commitment, encouraged by the project's advisory board, served a form of motivating certain underrepresented fields to feel addressed and motivated to bring their perspective into the discourse.

Overall, the reflection in the self-evaluation of the project showed that, for the phase of stakeholder identification, the securing of transparency of the selection of participants (especially regarding organizations with potential conflicts of interest such as, for example, the pharmaceutical industry or private insurers), the insuring of balance of positions and results, the address of local levels of dialogue, and the sharing and dissemination of results to connect stakeholders were declared aims in the entire discourse procedure. It was set out that any declarations of bias should be required from all participants to avoid potential conflicts of interest. At the beginning of the project, the project team hesitated regarding the decision on whether to invite government organizations. However, due to the fact that our project was funded by the government, this affected our decision to invite governmental bodies. This could be very different for discourses with other sources of funding.

\section{3) Representativeness}


From the beginning of the discourse, it seemed inevitable to engage a heterogenous compilation of representatives to be able to formulate accurate recommendations, especially based on the topics addressed and discussed in the position papers as well as in the joint statement. The project team decided not to include individual experts as far as possible to strengthen legitimacy. However, while good representation can help strengthen legitimacy, it does not guarantee it.

In a few position papers, the procedural step of engaging various stakeholders was highlighted to jointly cooperate and develop best practices as well as standards for the field of diagnostics and for the validation of biomarkers. In several positions papers, organizations referred to the multidisciplinarity and interdisciplinary networking in their own organization or to multidisciplinarity being a driving factor in their organization's policy work. This was linked to their expectations of the working environment and approach of the discourse at hand. Further, organizations described certain attributes in the position papers including their work in a competent, comprehensive, and multidisciplinary manner to the needs of those affected.

Assessment in the position paper, the joint statement, and the commentaries by stakeholders show that the multidisciplinary composition of stakeholders was appreciated and commended. Especially, in the majority of the evaluation forms, the diversity and combination of perspectives as well as the interdisciplinary exchange and approach were perceived very positively. Others highlighted the respect of minority opinions in the process and the opportunity of good networking and exchange across fields. However, in one evaluation form it was mentioned that some representatives of associations ultimately did not act as representatives but acted as individual expert representatives. According to formal documentation including the position papers, the joint statement, project steps, protocols of project meetings, however, only two people actually took part in the stakeholder conference who wanted to be considered "individuals" and not as representatives of the organization they worked for.

Looking more closely at the aspect of representation, there were some perspectives that were missing in the discourse. As reflected in the self-evaluation of the project, the perspectives of health economics and the general practitioners were missing in the first phase of the obtained written statements. During the conference, there was no personal representation of patient advocacy due to last-minute cancellation because of illness. According to the self-evaluation of the project, it would have been desirable for these perspectives to be represented more prominently in the discourse. In the end, it was possible to methodically introduce the perspective of those affected in the further procedure. The detailed position paper of the German Alzheimer's Society found resonance in the joint statement and subsequently the German Alzheimer's Society and the German Society for General and Family Medicine expressed their explicit support for the joint statement in the commentary phase. Nevertheless, the inclusion of the health economic perspectives remains an important task for future discourse processes on the issue at hand as the financing of predictive biomarkers was a crucial issue in the joint statement. In the discourse, organizations focusing on the health economic perspective did not show interest in participation. 
Further, as mentioned above, none of the organizations invited who had a government mandate actively participated in the discourse. With the exception of the Federal Ministry of Family Affairs, Senior Citizens, Women and Youth which did not actively take part in the stakeholder conference but took a clear stance towards the discourse project. It outlined its positive expectations towards the output of recommendations that should serve as a stepping stone for further measures to be taken. In the phase leading up to the stakeholder conference, the Ministry applauded the project's accomplishment due to the wide-ranging selection of stakeholders and representation as well as the members of the project's advisory board. The letter also referred to the ministers working on the German national dementia strategy as one of the stepping stones for dissemination.

As taken from the self-evaluation of the project, the project team was very satisfied with the breadth of interests represented in the discourse process. Also, newspaper reports commented on the heterogenous composition of stakeholders during the conference and as authors of the joint statement. Further, the advisory board in its project commentary valued that the project managed to reach a wide spectrum of actors.

The project design had features of useful redundancy, allowing multiple entry points for organizations to engage in the discourse according to organizations readiness and ability (see Figure 1). With time, the project became more well-known through media coverage and a snowball principle. This had the effect that organizations saw the need to also participate and bring their perspective into the discourse.

\section{4) Process management}

The material of the project steps, the protocols of project meetings, and the self-evaluation of the project showed that the time frame of the discourse procedure was optimized with the incorporation of participatory phases; the encouraged position papers led to an early discussion of the organizations with the topic which enabled a nuanced but goal-oriented discussion at the conference itself. The establishment of thematic working groups at the conference helped structure and make the discussion and joint writing possible in the available time frame.

From the project team's perspective, much effort was invested in setting of deadlines, communication, and sending of reminders to reach project milestones (see Figure 1). This included the initial step of inviting identified stakeholders to take part in the discourse with a position paper and committing to attendance at the stakeholder conference as well as in the editing phases and the commentary phase. A strict timeline and time slots between the different participatory phases made it possible to achieve such a process in the dedicated time frame. Regular meetings of the project team allowed for concise planning and also revision of planning. The process management was made possible with two full project positions over the extent of two years.

Regarding the stage of research, the importance of engaging on a discourse early on for a topic that has not manifested its implications in practice yet, was stressed in the self-evaluation of the project as well as 
by participating stakeholders in the evaluation forms. As can be taken from the evaluation forms, the majority of stakeholders felt well informed about the project at all times and in all evaluation forms, stakeholders expressed that they had received adequate information in preparation for the stakeholder conference. Timely provision of information was a crucial aspect determining the successful implementation of the discourse.

The explicit comments on a procedural level focused on the difficulty given the complexity of the topic and the wish to have more time to specify statements and definitions of terms. Also, in the commentary by advisory board, the advisory board emphasized that the stakeholder conference and the result of the joint statement highlighted that further discourses are needed to address unresolved issues. Thus, the initiated discourse, can be regarded as a successful starting point for further discourses.

\section{5) Output}

\section{References to thematic output}

The material, especially the project steps, protocols of project meetings, and the self-evaluation of the project showed that the overarching aim of the stakeholder conference was to achieve a consensus statement. The second aim was to make the landscape of interests and demands transparent and to define the frame of reference, in which the discourse procedure can take place.

From the protocols of project meetings, it became evident that at all times, the project team took queries, criticism, and praise seriously. Regarding the procedure, numerous loops of more clearly defining information leading up to the stakeholder conference were taken to address the issues put forward by participating stakeholders.

The positions papers included a concrete discussion of the critical aspects of dementia prediction from their organization's perspective which made it possible to efficiently frame the topics to be discussed at the stakeholder conference. First, organizations were encouraged to think about and deal with the topic in advance. Second, this allowed the project team to prepare adequately by condensing the 29 position papers' content and preparing three thematic working groups. The positions papers were sent to all conference participants in advance to enable a form of exchange before the actual conference.

Regarding the timely addressing of the issue at hand, many position papers addressed topics that needed clarification before the medical technology was actually applied in clinical practice. For the patientrelevant use of biomarkers, it was further put forward by the participating stakeholders in the position papers that the arising ethical aspects and arguments should be revealed transparently and systematically as well as transported to other stakeholders to allow for a comprehensive assessment of the technology. This could also apply to the entire discourse at hand.

As becomes evident from the scope of the position papers, the joint statement and also the commentaries by stakeholders as well as the scientific publications and reports on the project, the 
discourse has accomplished an analysis of the topic put up for discussion which, however, is not concluded in its entirety. The discourse was able to spark discussion and attention and also to develop crucial areas relevant to health policy, restructuring of counseling, and public perception or dealing with dementia. Certain issues were touched upon but not resolved which concurs with the initially set out aim of the discourse procedure. Numerous topical points were addressed in the joint statement with the need of further exploration and discussion regarding concrete implementation.

Also perceived limitations to what the discourse or at least the stakeholder conference could accomplish were formulated in some of the commentaries by stakeholders, taking up points that should be further discussed in a societal discourse. As can be taken from one interview, sustainable solutions are more likely to emerge from open discourses than from leaving such discussions to individual experts.

\section{References to appreciation and support of the discourse}

In some of the positions papers, the author(s)/organization expressed gratitude for being able to take part in the discourse or and in several positions papers the discourse approach with a wide discussion was actively welcomed.

In other position papers, support of dealing with the topic of dementia prediction was expressed and participation of specific organizations was recommended that were found imperative to include such as the German Alzheimer's Society and the German Ethics Council. Further, some organizations stressed why it was important that they themselves were included in the discourse.

The advisory board in their commentary on the entire discourse procedure expressed praise over the project's thorough and critical approach. The commentary reflected the interaction and active participation in the project. The advisory board applauded the highly ambitious aim of reaching potentially all relevant stakeholders in Germany with the discourse and added that the success of the discourse was not guaranteed.

Overall, the evaluation forms by stakeholders show that the stakeholder participants were satisfied with the stakeholder conference and the engagement process. Further, in all EFs stakeholders stated that they would take part in such a discourse on medical-ethical topic again.

\section{References to expectations and demands}

In the SPs, expectations to the process were raised regarding the need for discussion on certain aspects of prediction that were missing up until the discourse was initiated or needed further attention and dealing with. In some of the SPs, the authors referred, on the one hand, to an inclusive and appreciative approach aimed at catering to the needs of those affected and, on the other hand, to an approach dealing with the topic of dementia prediction as such. This allows to make inferences about the expectation of identifying such attitudes in the discourse process itself. 
The Federal Ministry of Family Affairs, Senior Citizens, Women and Youth, as mentioned above, did not actively take part in the discourse but took a stance towards the discourse project and outlined their expectations on the discourse in a letter. The Federal Ministry welcomed the initiated discourse through the German Federal government and described its expectations towards the output of recommendations that should serve as a stepping stone for further measures to be taken and applauded its success due to the wide-ranging selection of stakeholders and the representation as well as the members of the project's advisory board.

Further, the letter referred to the ministers working on the German national dementia strategy and the possibility of linking outcomes.

In a SR, the project aims were described as to stimulate a social discourse and accompany the process of medical progress. In a few cases, organizations used the format of the SPs to demand certain professions be equipped with more competencies from which people with dementia could potentially profit and also specifically regarding biomarker tests.

\section{Dissemination of output}

The evaluation forms by stakeholders state that the majority of stakeholders wanted to introduce certain objectives into the procedure. Further, in the majority of the valuation forms it was stated that the stakeholders were able to gather new information that is relevant for their field of work through the discourse including specific knowledge about the issue at hand, perspectives from other professions, and processes of health policy work. The interest in and perceived importance of moving the discussions of the stakeholder conference forward were strongly expressed in the evaluation forms highlighting ideas for dissemination of the results of the stakeholder discourse.

As can be taken from the self-evaluation of the project, the project team pursued the goal of producing a joint consensus statement with the initiation of the engagement process and specifically with the stakeholder conference. The chosen methodology in thematic working groups during the stakeholder conference took into account the plurality of perspectives. The participants were able to identify and clearly state areas of consent and also of dissent at any time. The commentary phase made it possible for a multitude of organizations to present or highlight points of consent and support or of dissent and controversy on the produced content of the joint statement. Especially controversial points could be presented with different arguments and in their plurality.

The results of the discourse were disseminated at various points in scientific conferences and at public project events such as a press conference and the final conference of the project. The project team always activated press representatives for important steps in the procedure contributing to a flow of dissemination during the entire approach. The project aims and results were taken up by multiple news agencies, organizations, and other institutions. Some of the participating organizations made an effort to present the project on their channels and beyond. Importantly, the project was mentioned in the report of 
the German National Dementia Strategy referring to the procedure as an important step for a societal discourse on dementia prediction.[2]

The project team reflected early on at the beginning of the project, which kind of follow-up project could be initiated. It became clear from the beginning that the focus should lie on counseling approaches and the development of guidelines.[3]

\section{6) Role definition}

The material shows only few references to role expectations of the discourse organizers and moderators. The advisory board made clear in its commentary, which was also described in the project steps, protocols of project meetings, and the self-evaluation of the project, that its primary purpose is to consult the project team ranging from the initial identification and selection of stakeholders to dissemination of the project results.

In the self-evaluation of the project, the project team states its task to transparently steer, moderate, bring together and reflect on the scientifically-based, result-oriented and participatory discourse and, if necessary, to modify or adjust project steps and participatory phases. On the content level, the project team is editorially responsible for obtaining representation of relevant organizations, all project results as well as for the formulation of ethically reflected recommendations for political decision-makers. Project reporting and other forms of publication also are declared as part of the project team's responsibility. Furthermore, the project team sees its responsibility in initiating follow-up research and supporting the continuation of the discourse (e.g. through dissemination, teaching, input into specialist discourses such as the German National Dementia Strategy).

According to the project steps, protocols of project meetings, and the self-evaluation of the project, the project team also sees it as its task to promote the discourse in the general public. For example, a population survey conducted by the AbbVie Healthcare Monitor placed a number of questions on dementia prediction in its survey. A further measure was social media engagement including Facebook and Twitter, enabling an exchange of discussions on questions the project team provided on the prediction of dementia and current information on the project. The general social media uptake can be considered moderate.

The role of the editing team of the joint statement was a politically sensitive task. The editing team was appointed by the stakeholders present at the conference with the assignment to finalize the joint statement without significant changes to consented essence of the composed statement to that point.

During the planning of and preparing for the discourse, it became an immense issue to reflect and define our own roles researchers and moderators of the discourse. As the project team, we were also accountable to the sponsor of the project which promised an output but at the same time needed to let stakeholders drive the content and consensus procedure. 


\section{7) Transparency}

Overall, the project team clearly documented all procedural steps. My research interest in evaluation was supportive of this. The underlying premise of the entire project based on disclosure of interest-driven exertion of influence and openness to the discourse as a condition of participation.

Regarding the stakes of participating organizations, the project team asked the authors of the position papers in the first question to make clear how or to which extent their organization dealt with issues of dementia and with this, elucidating their stake in the discourse. Participating organizations specified in which form they approached the topic of dementia and people affected by dementia including relatives and partners.

Further, by inviting organizations to answer questions and send the position papers in advance to the stakeholder conference, the participating organizations dealt with the topics from their organization's perspective. All position papers addressed issues that were later taken up during the conference. Although some papers may have indulged in the topic in a deeper fashion, all organizations in advance positioned themselves towards the issue put forward in the discourse approach.

Regarding the procedure leading up to the stakeholder conference, it can be taken from the project steps, protocols of project meetings and the self-evaluation of the project that all material and information relevant to participating organizations prior to the stakeholder conference as well as after including all position papers, the joint statement and the commentaries by stakeholders was made publicly available on the project's website striving for procedural and content-related transparency.

However, in the evaluation forms by stakeholders, it became evident that some information provided in preparation for the stakeholder conference and at the beginning of the conference was missing for some stakeholders. This mainly referred to definitions of terms distinct to the topic of dementia prediction, for example, the term 'prediction' or that the target group for the clinical application of blood-based biomarkers included people with early symptoms of cognitive impairment but also asymptomatic people. We as the project team should have been more precise with definitions and should have consulted experts on these in advance.

For the production of a joint statement, the working groups served to produce topical texts, the plenary sessions were used to reach a form of consensus on the results while at the same time identifying and denominating areas of dissent. Directly after the stakeholder conference the content of the joint statement was presented at a public panel discussion at which also Lower Saxony's Minister for Social Affairs, Health and Equality took part.

However, regarding transparency of the editing procedure of the joint statement, one organization in the commentary phase used its commentary to highly criticize that changes were made to the consensusversion by the editing team that strongly went against this organization's perspectives. The issue 
regarding specific formulations in the joint statement was resolved in an additional round of editing and was made transparent in the discourse.

Following the stakeholder conference, all stakeholders were invited by the project team to comment on the joint statement. This commenting phase was opened not only to the stakeholders who participated during the stakeholder conference, but to all organizations originally identified and invited to take part in the discourse. This procedure, supported by the project advisory board, made it possible to present or highlight points of consent and support or of dissent and controversy in retrospect. Especially controversial points could be presented with different arguments and in their plurality.

As can be taken from the self-evaluation of the project, the discourse procedure, which guided heterogeneously acting and thinking stakeholders to agree on a common position, reflects both valueoriented interests (e.g. protective standards) as well as particular interests (e.g. naming responsibilities for counseling processes and further health-policy interests). This can partially be explained by the fact that the discourse included organizations representing particular interests due to the professional background and mission of the organization. None of the organizations participating at the stakeholder conference had a government mandate to mediate interests. For external readers, it must therefore be communicated transparently which stakeholders were involved in the joint statement. Due to the participating stakeholders' heterogeneity and solution orientation, they were able to find overarching value-oriented statements. From the project team's perspective, this is an indicator for the qualitative added value of this method, namely to sound out concrete consensus on standards. The procedure including the editing in the aftermath of the stakeholder conference and also the commentary phase, required much coordination, allowed for further consensus and support to be reached.

\section{Discussion}

My analysis indicates the key facilitating elements and also barriers of this participatory approach, demonstrating the importance of especially deploying the following evaluation criteria: output, representativeness, process management, and transparency. Overall, regarding the outcome and selfreflection of the discourse procedure outlined in the corpus of material, key facilitating elements for the stakeholder approach's success include the meticulous and transparent documentation and provision of information, the reiterative "relationship-building" to secure participation of stakeholders as well as the active response to feedback during the entire discourse allowing for a discursive approach which other processes have also highlighted (Laird et al. 2020; Boaz et al. 2018).

The analysis also showed that key barriers of the stakeholder approach included issues of finalizing the joint statement attributed to limited time during the stakeholder conference as well as the subsequent need for an editing phase and editing team. Further barriers were issues of agreeing on a consensus. Barriers were also posed by one stakeholder's criticism of transparency as well as the absence of certain groups throughout the discourse and also at decisive points of the discourse procedure. These main findings will be discussed in a more nuanced manner in the following section. 


\section{Added value of seven evaluation criteria}

Other studies have proposed the need for systematic evaluation of stakeholder approaches (e.g. Ray \& Miller 2017; Lemke \& Harris-Wai 2015). My methodological reflection aims at, on the one hand, building on developed approaches and frameworks of stakeholder identification and engagement design (especially Boaz et al. 2018; Ray \& Miller 2017; Manafo et al. 2018; Concannon et al. 2014) and, on the other hand, learning from limitations and shortcomings of previous approaches and supplementing useful criteria for future application. These seven developed normative evaluation criteria have been effective for the evaluation of the stakeholder discourse on dementia prediction. These evaluation criteria may also be conducive to the evaluation of engagement processes on other health research topics with a large corpus of material.

From the analysis of the corpus of material, one criterion attracts attention as one that has not been applied as an established evaluation criterion in previous work, namely, output. Other criteria applied for the analysis in this procedure match previous findings to some extent, but no approach, to my knowledge, has incorporated such an elaborate composition of normative evaluation criteria.

Regarding the criterion output or more specifically, the determination of disseminating project output, there was a pronounced interest of the participating stakeholders in disseminating the results.

Dissemination was attained over the organizations' platforms, media, publications, and discussions in professional associations. This highlights the stakeholders' investment in the discourse and the topic discussed. As Manafo et al. (2018) and Ray \& Miller (2017) have pointed out, evaluation frameworks as well as sufficient data is needed as a basis for evaluation to measure not only immediate outcomes but also intermediate and long-term outcomes of an engagement processes. These are relevant for the context of patient as well as expert stakeholder engagement. The advance of effective and sustainable engagement processes relies on documented outcomes of the respective procedure (Manafo et al. 2018; Ray \& Miller 2017).

On a practical level, stakeholder engagement identifies areas of agreement as well as disagreement and provides an opportunity to understand more fully what might be driving differences in stakeholder interests. Stakeholder input may also help articulate the values of the broader community affected and align policy recommendations with these expectations (Lemke \& Harris-Wai 2015). As common in deliberative engagement (Murtagh et al. 2017), our main content output was the production of a joint statement including recommendations on the issue under discussion. Regarding the production of a joint outcome, consensus was encouraged to support a policy-oriented outcome, but was not required. Dissenting perspectives and minority positions were also included in the joint statement and found further resonance in the commentaries.

As set out, engagement aims to be democratic, empowering, and educational. Thus, with the criterion output, it was possible to highlight the gratifying notion that the participating stakeholders learned new information that is relevant for their field of work through the engagement process. 
Further, in the case study at hand, it became evident from the evaluation that representativeness is an important criterion. While good representation helps strengthen legitimacy, it does not guarantee it. Organizers of discourses are responsible for enabling representation. The absence of patient advocacy groups, health economics and governmental bodies may affect the perceived legitimacy as well as sustainability of such an approach. The decision becomes relevant of who should participate in a discourse on novel issues or innovations adjacent to a common topic. The potential degree of polarization between stakeholders should be critically reflected the development of consensus for policy development is aimed at (MacLean \& Burgess 2010). Especially regarding the topic of dementia, due to the aspect of cognitive impairment which has in the past stood as a contrasting pole towards political participation (e.g. research participation; see, for example, Jongsma et al. 2016), the examination of and participation in the discussion on dementia prediction can serve as a window of opportunity to break that traditional conception apart. In participatory approaches involving stakeholders, it has become a common practice to revert to already established groups, for example, established advocacy groups. For the particular case study at hand, the question arises whether established advocacy groups in the context of dementia and dementia care suffice to represent issues of dementia prediction. The argument can be made that affected groups for the particular topic of dementia prediction are not well represented or that advocacy does not yet exist in this context. From a methodological and evaluation perspective, representation raises two critical questions: Does the inferential process in a particular setting actually require direct representativeness? And even if it is inferentially desirable can representativeness in the sense representing perspectives of patients or affected people (Gerhards et al. 2017), in practice, be achieved? While engagement processes generally aim at being representative and inclusive, these questions need to be raised according to the topic being discussed as well as the envisioned policy outcome (Slack et al. 2018; Murtagh et al. 2017).

Further, the criteria, process management, role definition, and transparency materialized as crucial elements for the evaluation of the engagement process. Especially regarding the aspects of planning and legitimacy of process outcomes, obligation and implementation played a pivotal role in making the engagement process possible. Potentially, the criterion of role definition could be even more relevant for engagement processes negotiating highly controversial topics, such as the use of data and information in biomedicine (Peek \& Rodrigues 2018) or the current topic of vaccine allocation (Wu et al. 2020). The criteria depth of participation and legitimate selection of stakeholders rather seem to function as preconditions for the effective implementation of an engagement process.

\section{Corpus of material for evaluation}

The developed normative evaluation criteria make the evaluation of an engagement process with a large amount of material possible. In the process of developing practical criteria, the importance of documentation of all project steps becomes clear if there is an authentic interest in evaluating an engagement process. The analysis of the discourse procedure used as a case study demonstrated the benefit of obtaining and collecting external evaluation but also internal documentation of project steps, 
e.g. summaries, protocols, and self-evaluation. The focus of evaluation often tends to be on external evaluation mirroring the experience and perspectives of those engaged in the process. However, with the sole focus on external evaluation, important procedural aspects of the entire engagement process will not be covered. Accordingly, with the application of the developed criteria, I stress the importance of including both external as well as internal material for the evaluation of engagement processes. The incorporation of both external and internal material supports taking the approach to evaluation seriously but also strengthens the option of refuting or reconsidering evaluation statements. Other studies have also highlighted the importance of detailed documentation of stakeholder engagement and the need for more standardized reporting guidelines (see for example Ray \& Miller 2017).

Taking into account the breadth of material in this case study, my analysis can be considered a form of participatory observation. While it can be assumed that internal material may gloss over procedural elements, the material at hand actually was more critical in pointing out missing perspectives or absence of governmental bodies in the engagement process.

\section{Conclusions}

While previous studies (e.g. Knoepke et al. 2019; Brown \& Bahri 2019; Boaz et al. 2018; Deverka et al. 2013) have demonstrated the benefits and the manifold application of engagement processes, evaluation and especially evaluation frameworks are currently missing for expert stakeholder engagement in the health care context. Evaluation can contribute to the comparability of different engagement processes, to the assessment of their respective output as well as the recognition of an initiated engagement process. Meaningful engagement processes tend to be long, work- and timeintensive and, to some extent, not controllable. Evaluation can further be helpful to assess strengths and potential pitfalls for the future planning of other engagement processes (Manafo et al. 2018, Boaz et al. 2018; Ray \& Miller 2017). Thoroughly documented evaluation can additionally contribute to strengthening engagement outcomes for the formulation of policies and, at the same time, disconfirming specific outcomes with little relevance for the scientific context or policy development. Especially in a country such as Germany with comparatively little experience in engagement processes the risk of skepticism can be countered with the application of thorough evaluation frameworks.

Existing evaluation frameworks of engagement processes focus mainly on patient engagement. While the proposed elements of evaluation can be applied for the evaluation of other engagement processes, expert engagement can be considered a particular case due to institutional or organizational resources they have. The resources experts rely on can be considered beneficial when the aim of an engagement process entails the negotiation of recommendations for new biotechnological approaches in the health care context. However, expert stakeholder engagement does not replace patient engagement. Expert engagement can be regarded as a first step of identifying relevant issues in a particular field. The advantage of including specialized experts and disciplines in a discourse can be mirrored in the potential of efficient development of recommendations and policy making. The disadvantage clearly lies in the 
potential of undermining vulnerable groups such as patient advocacy groups and other lay stakeholder groups which are not represented within an organization.

Provided the engagement process is encompassing enough, expert engagement can later on be interlinked with patient engagement to address particular necessities of this group which may not be covered by expert engagement. The addition of lay stakeholders is especially inevitable and of utmost importance for the discussion of bioethical issues that affect society as a whole. As conveyed at the outset of this paper, PPI is considered imperative for the identification of relevant research and its implementation. Considering the case study at hand on dementia prediction, the negotiation of the topic is relevant to affected people, the entire health care system as well as society as a whole. Patient advocacy can be considered a starting point for including relevant stakeholders. However, for the topic of dementia prediction patient advocacy can be considered too particular, because new developments in blood-based biomarkers will not only deeply affect patients and the care system but also healthy or asymptomatic people who can potentially be tested.

It becomes clear in the entire corpus of material on the discourse project that a research project cannot replace the societal discourse. It can act as an initiator and can offer methodological structuring recommendations. This shows the importance of defining what such a discourse project can and wants to achieve and what the limitations are. If the focus lies on policy development, the importance may arise to include governmental organizations in the engagement process. For this, the recommendation for future engagement processes is to be more persistent on why an organization decides not to participate.

In our case, the educational approach can be expanded, political committees can be triggered to further assess practical implications and with the follow-up project we actually fulfilled our aim. While these evaluation criteria can also be used for shaping and planning of engagement processes, specific guidelines for the development and implementation of stakeholder processes should be consulted in detail (e.g. Petkovic et al. 2020).

\section{Limitations}

Regarding the corpus of material, some of the material, which is marked accordingly in Figure 1, is selfevaluatory and produced by our project team. My role in the research project was to carry co-organize the stakeholder engagement process on a content and organizational level. This including the phase of evaluation. For the methodological reflection of the engagement process, it was important for the research project to be finalized, so that it had no effect on the project's final report for the sponsor of the project.

This paper is to be regarded as an explorative approach rather than a quantitative assessment of a discourse procedure on dementia prediction. The case study examining the advantages but also disadvantages in this approach can contribute to future projects in this field, however, it is not representative for all engagement processes. 
A content-related paper will be published separately. In other cases, the content as such and its assessment can be regarded as an important indicator or criteria for evaluating a procedure.

\section{Declarations}

\section{Acknowledgements:}

I would like to thank the entire research team including Silke Schicktanz, Scott Stock Gissendanner and Benjamin Herten for their productive collaboration on the research project and the output created. I also would like to thank the stakeholders who took part in the engagement process. I especially want to thank Silke Schicktanz and Scott Stock Gissendanner for their insightful comments on and support with this manuscript.

The project: "Dilemmas of Predictive Dementia Diagnostics: German Stakeholder Conference for Improving Ethics Competence in Healthcare and Life Sciences" (October 2017 - April 2020) was funded by the German Federal Ministry of Education and Research (BMBF); grant no.: 01GP1770A+B.

The funding body had no role in the design of the project, analysis, and interpretation of data and in writing the manuscript.

\section{References}

1. Adashi EY, Cohen IG, Hanna JH, Surani AM, Hayashi K. Stem Cell-Derived Human Gametes: The Public Engagement Imperative. Trends Mol Med. 2019;25(3):165-167.

2. Alpinar-Sencan Z, Lohmeyer L, Schicktanz S. Planning Later Life with Dementia: Comparing Family Caregivers' Perspective on Biomarkers with Laypersons' Attitudes towards Genetic Testing of Dementia Prediction. New Genet Soc. 2019; doi:10.1080/14636778.2019.1637719.

3. Archana S, Karmacharya B M, Rashmi M, Abhinav V, Meghnath D, Natalia O, Rajeev S, Prajjwal P, Annette F, David C, Swornim B, Roman XD, Donna S, Rajendra K. Stakeholder Engagement in Planning the Design of a National Needs Assessment for Cardiovascular Disease Prevention and Management in Nepal. Glob Heart. 2019;14(2):181-189.

4. Atkins CGK. The Limits of Liberal Values in the Moral Assessment of Genomic and Technological Innovation. In: O'Doherty K, Einsiedel E, editors. Public Engagement and Emerging Technologies. Vancouver, London: UBC Press; p. 310-321.

5. Balane MA, Palafox B, Palileo-Villanueva LM, McKee M, Balabanova D. Enhancing the use of stakeholder analysis for policy implementation research: towards a novel framing and operationalised measures. BMJ Global Health. 2020;5:e002661.

6. Barfoot J, Doherty K, Blackburn CC. EuroStemCell: A European infrastructure for communication and engagement with stem cell research. Semin Cell Dev Biol. 2017;70:26-37. 
7. Beier K, Jordan I, Wiesemann C, Schicktanz S. Understanding collective agency in bioethics. Med Health Care Philos. 2016;19(3):411-422.

8. Boaz A, Hanney S, Borst R, O'Shea A, Kok M (2018). How to engage stakeholders in research: design principles to support improvement. Health Res Policy Syst. 2018;16:60.

9. Brown P, Bahri P. 'Engagement' of patients and healthcare professionals in regulatory pharmacovigilance: establishing a conceptual and methodological framework. Eur J Clin Pharmacol. 2019;75(9):1181-1192.

10. Camelo Castillo W, Heath N, Kim J, Yang K, Ritchey ME, dosReis S, Santanello N, West SL (2019). Engaging stakeholders in pharmacoepidemiology research: Current state and recommendations. Pharmacoepidemiol Drug Saf. 2019;28(6):766-776.

11. Concannon TW, Fuster M, Saunders T, Patel K, Wong JB, Leslie LK, Lau, J. A systematic review of stakeholder engagement in comparative effectiveness and patient-centered outcomes research. $\mathrm{J}$ Gen Intern Med. 2014;29(12):1692-1701.

12. Deverka PA, Lavallee DC, Desai PJ, Esmail LC, Ramsey SD, Veenstra DL, Tunis SR. Stakeholder participation in comparative effectiveness research: defining a framework for effective engagement. J Comp Eff Res. 2012;1(2):181-194.

13. Forsythe LP, Frank LB, Hemphill R, Tafari AT, Szydlowski V, Lauer M, Goertz C, Clauser S. (2018). Researchers, Patients, and Stakeholders Evaluating Comparative-Effectiveness Research: A MixedMethods Study of the PCORI Reviewer Experience. Value Health. 2018;21(10):1161-1167.

14. Gesell SB, Halladay JR, Mettam LH, Sissine ME, Staplefoote-Boynton BL, Duncan PW. Using REDCap to track stakeholder engagement: A time-saving tool for PCORI-funded studies. J Clin Transl Sci. 2020;4(2):108-114.

15. Gesell SB, Klein KP, Halladay J, Bettger JP, Freburger J, Cummings DM, Lutz BJ, Coleman S, Bushnell C, Rosamond W, Duncan PW (2017). Methods guiding stakeholder engagement in planning a pragmatic study on changing stroke systems of care. J Clin Transl Sci. 2017;1(2):121-128.

16. Glymour MM, Brickman AM, Kivimaki M, Mayeda ER, Chêne G, Dufoudil C, Manly JJ. Will biomarkerbased diagnosis of Alzheimer's disease maximize scientific progress? Evaluating proposed diagnostic criteria. Eur J Epidemiol. 2018;33(7):607-612.

17. Goodman MS, Sanders Thompson VL. The science of stakeholder engagement in research: classification, implementation, and evaluation. Transl Behav Med. 2017;7(3):486-491.

18. Hansen SL, Holetzek T, Heyder C, Wiesemann C. Stakeholder-Beteiligung in der klinischen Forschung: eine ethische Analyse. Ethik Med. 2018;30:289-305.

19. Hermans FLP, Haarmann WMF, Dagevos JFLMM. Evaluation of stakeholder participation in monitoring regional sustainable development. Reg Environ Change. 2011;11:805-815.

20. Hsieh HF, Shannon SE (2005). Three approaches to qualitative content analysis. Qual Health Res. 2005;15(9):1277-1288.

21. Jongsma KR, van Bruchem-Visser RL, van de Vathorst S, Mattace Raso FU (2016). Has dementia research lost its sense of reality? A descriptive analysis of eligibility criteria of Dutch dementia 
research protocols. Neth J Med. 2016;74(5):201-209.

22. Kendall C, Fitzgerald M, Kang RS, Wong ST, Katz A, Fortin M, Dionne E, Kuluski K, O'Brien MA, Ploeg J, Crowe L, Liddy C (2018). "Still learning and evolving in our approaches": patient and stakeholder engagement among Canadian community-based primary health care researchers. Res Involv Engagem. 2018;4:

23. Knoepke CE, Ingle MP, Matlock DD, Brownson RC, Glasgow, RE (2019). Dissemination and stakeholder engagement practices among dissemination \& implementation scientists: Results from an online survey. PLOS ONE. 2019;14(11):e0216971.

24. Laird Y, Manner J, Baldwin L, Hunter R, McAteer J, Rodgers S, Williamson C, Jepson R. Stakeholders' experiences of the public health research process: time to change the system?. Health Res Policy Sys. 2020;18:83.

25. Leibing A, Schicktanz S. Preventing Dementia? Critical Perspectives on a New Paradigm of Preparing for Old Age. New York, Oxford; Berghahn Books;

26. Lemke AA, Harris-Wai JN (2015). Stakeholder engagement in policy development: challenges and opportunities for human genomics. Genet Med. 2015;17(12):949-957.

27. MacLean S, Burgess MM. In the public interest: assessing expert and stakeholder influence in public deliberation about biobanks. Public Untersantd Sci. 2010;19(4):486-496.

28. Manafo E, Petermann L, Mason-Lai P, Vandall-Walker V. Patient engagement in Canada: a scoping review of the 'how' and 'what' of patient engagement in health research. Health Res Policy Syst. 2018;16:5.

29. Márquez F, Yassa MA. Neuroimaging Biomarkers for Alzheimer's Disease. Mol Neurodegener. 2019;14:21.

30. Milne R, Diaz A, Badger S, Bunnik E, Fauria K, Wells K. At, with and beyond risk: expectations of living with the possibility of future dementia. Sociol Health IIIn. 2018;40:969-987.

31. Mozersky J, Sankar P, Harkins K, Hachey S, Karlawish J. Comprehension of an Elevated Amyloid Positron Emission Tomography Biomarker Result by Cognitively Normal Older Adults. JAMA Neurol. 2018;75(1):44-50.

32. Murtagh MJ, Minion JT, Turner A, Wilson RC, Blell M, Ochieng C, Murtagh B, Roberts S, Butters OW, Burton PR. The ECOUTER methodology for stakeholder engagement in translational research. BMC Med Ethics. 2017;18:24.

33. Nabers A, Hafermann H, Wiltfang J, Gerwert K. A $\beta$ and tau structure-based biomarkers for a bloodand CSF-based two-step recruitment strategy to identify patients with dementia due to Alzheimer's disease. Alzheimers Dement (Amst). 2019;11:257-63.

34. O'Doherty KC, Burgess MM. Public deliberation to develop ethical norms and inform policy for biobanks: Lessons learnt and challenges remaining. Research Ethics. 2013;9(2):55-77.

35. Petkovic J, Riddle A, Akl EA, Khabsa J, Lytvyn L, Atwere P, Campbell P, Chalkidou K, Chang S M, Crowe S, Dans L, Jardali FE, Ghersi D, Graham ID, Grant S, Greer-Smith R, Guise JM, Hazlewood G, Jull J, 
Katikireddi SV, ... Tugwell, P. Protocol for the development of guidance for stakeholder engagement in health and healthcare guideline development and implementation. Syst Rev. 2020;9:21.

36. Pii, KH, Schou LH, Piil K, Jarden M. Current trends in patient and public involvement in cancer research: A systematic review. Health Expect. 2019;22(1):3-20.

37. Pollock A, Campbell P, Struthers C, Synnot A, Nunn J, Hill S, Goodare H, Morris J, Watts C, Morley R. Stakeholder involvement in systematic reviews: a scoping review. Syst Rev. 2018;7:

38. Ray KN, Miller E. Strengthening stakeholder-engaged research and research on stakeholder engagement. J Comp Eff Res. 2017;6(4):375-389.

39. Renz SM, Carrington JM, Badger TA. Two Strategies for Qualitative Content Analysis: An Intramethod Approach to Triangulation. Qual Health Res. 2018;28(5):824-831.

40. Sacristán JA, Aguarón A, Avendaño-Solá C, Garrido P, Carrión J, Gutiérrez A, Kroes R, Flores A. Patient involvement in clinical research: why, when, and how. Patient Pref Adherence. 2016;10:631-640.

41. Schicktanz S. Politikberatung im Kontext der Medizin. In: Bröchler S, Schützeichel R, editors. Politikberatung, Stuttgart: Lucius \& Lucius UTB; 2008. p. 47-69.

42. Schicktanz $S$ (2015). The ethical legitimacy of patient organizations' involvement in politics and knowledge production: epistemic justice as conceptual basis. In: Wehling P, Viehöder W, Koenen S, editors. The public shaping of medical research, London: Routledge; 2015. p. 246-265.

43. Schiller C, Winters M, Hanson HM, Ashe MC (2013). A framework for stakeholder identification in concept mapping and health research: a novel process and its application to older adult mobility and the built environment. BMC Public Health. 13;428.

44. Schmitz-Luhn B, Jessen F, Woopen C. Alzheimer-Demenz: Biomarker zur Risikoprädiktion. Dtsch Arztebl. 2019;116(37): A-1592 / B-1315 / C-1294

45. Slack C, Wilkinson A, Salzwedel I, Ndebele P (2018). Strengthening stakeholder engagement through ethics review in biomedical HIV prevention trials: opportunities and complexities. J Int AIDS Soc 2018; 21 Suppl 7, e25172.

46. Souliotis K (2016). Patient participation in contemporary health care: promoting a versatile patient role. Health Expect. 2016;19(2):175-178.

47. World Health Organization: Global action plan on the public health response to dementia 2017 2025. https://apps.who.int/iris/bitstream/handle/10665/259615/9789241513487-

eng.pdf;jsessionid=8B9AE62F420034324ECE8ED9F7883CC0? sequence $=1$ (2017). Accessed 9 July 2020.

48. Whitty JA. An international survey of the public engagement practices of health technology assessment organizations. Value Health. 2013;16:155-163.

\section{Figures}




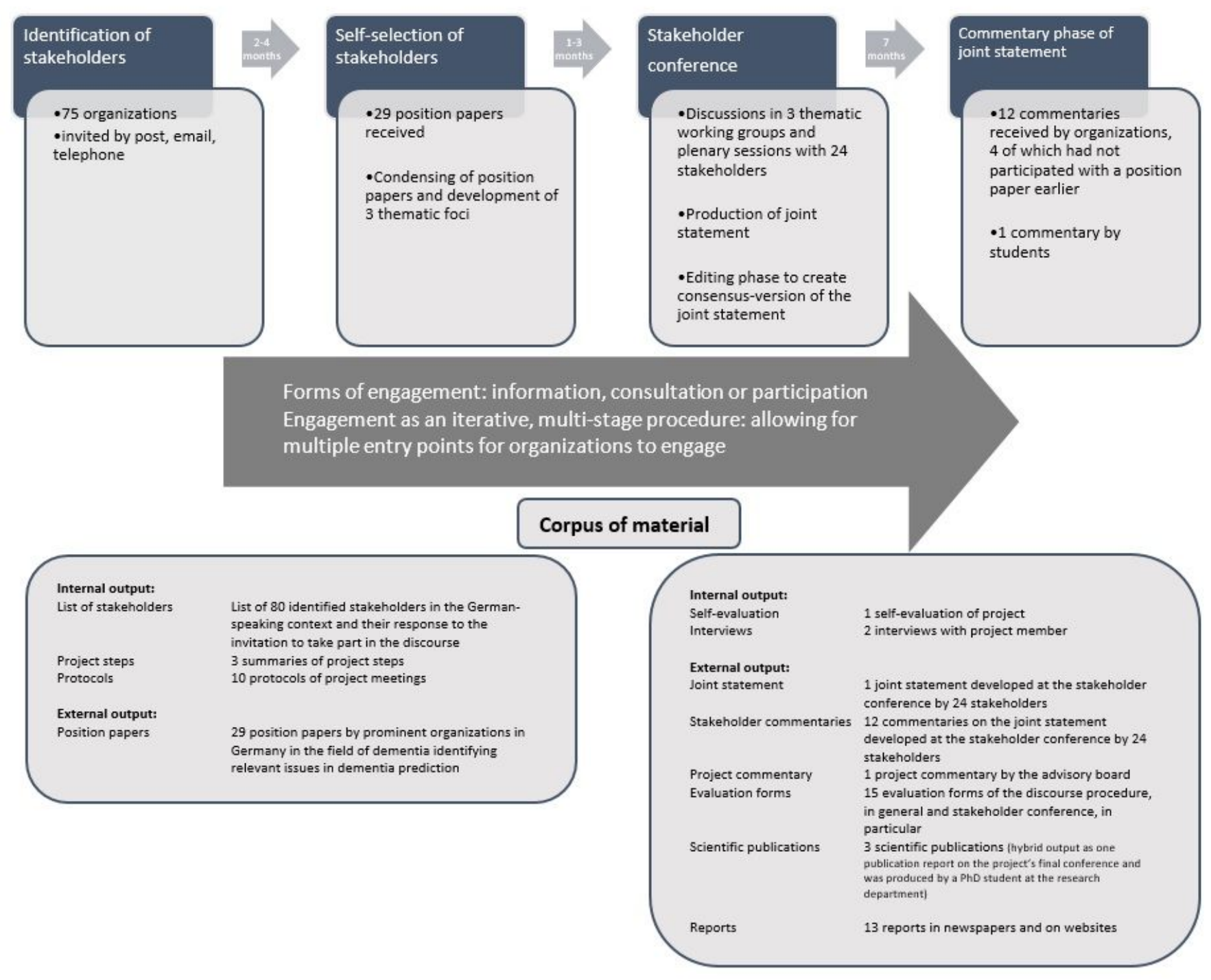

Figure 1

Phases of engagement (based on Brown \& Bahri 2019) 


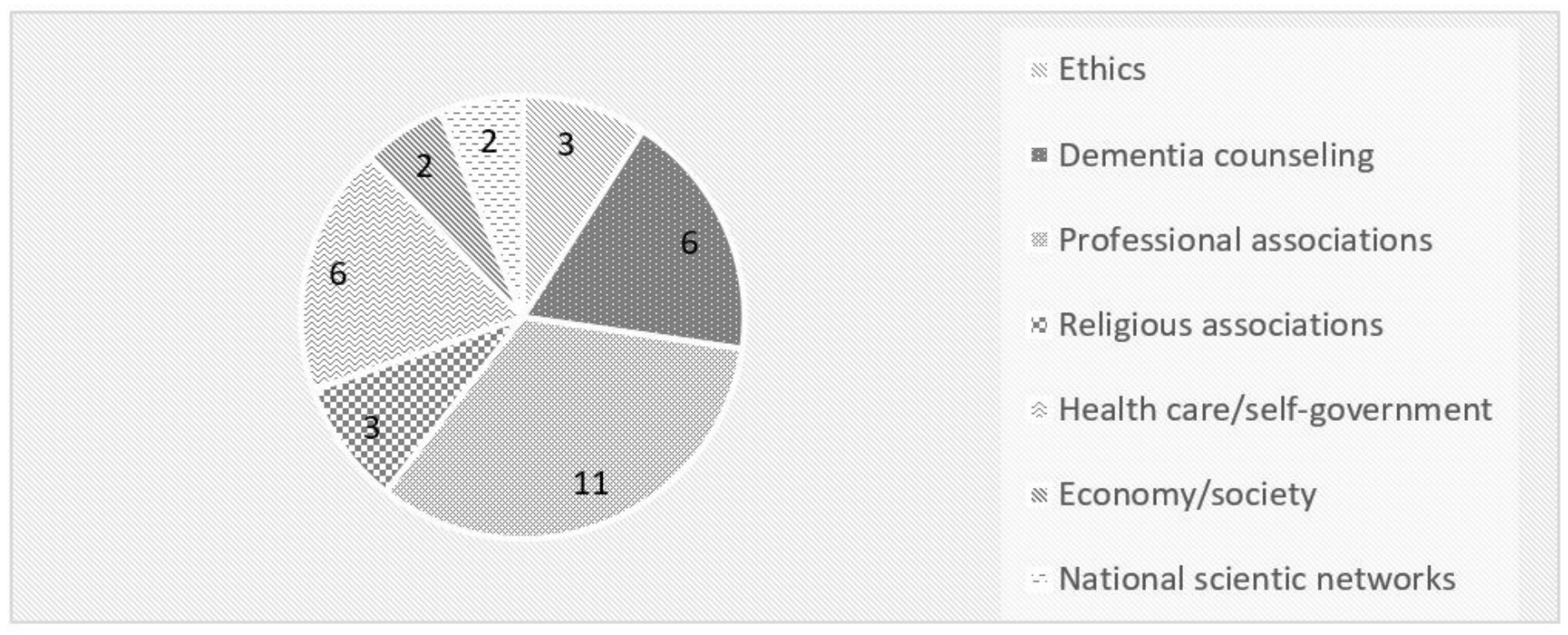

\section{Figure 2}

Fields of participating organizations and associations

\section{Supplementary Files}

This is a list of supplementary files associated with this preprint. Click to download.

- SupplementalMaterial1.docx 